\title{
Représenter « avec » le rite : réflexivité et danse rituelle dans un tableau murui-muina
}

Representing "with" ritual: reflexivity and ritual dance in a murui-muina painting

Representando "con" el rito: reflexividad y danza ritual en una pintura muruimuina

\section{Oscar Ivan Garcia}

\section{OpenEdition}

Journals

Édition électronique

URL : https://journals.openedition.org/jsa/19435

DOI : 10.4000/jsa. 19435

ISSN : $1957-7842$

\section{Éditeur}

Société des américanistes

Édition imprimée

Date de publication : 30 septembre 2021

Pagination : $77-102$

ISSN : 0037-9174

Référence électronique

Oscar Ivan Garcia, «Représenter « avec » le rite : réflexivité et danse rituelle dans un tableau muruimuina », Journal de la Société des américanistes [En ligne], 107-1 | 2021, mis en ligne le 30 septembre 2021, consulté le 02 septembre 2022. URL : http://journals.openedition.org/jsa/19435; DOI : https:// doi.org/10.4000/jsa. 19435 


\title{
Représenter « avec» le rite: réflexivité et danse rituelle dans un tableau murui-muina
}

\author{
Oscar Ivan GARCIA *
}

En analysant un tableau du peintre amazonien Brus Rubio Churay consacré aux bals rituels murui-muina, je montrerai le lien particulier que l'artiste établit entre l'art et le rituel: sa toile est moins une représentation du rituel qu'une représentation « avec» le rituel. En s'inspirant de la logique des bals rituels murui-muina, l'artiste crée un effet de « mise en abyme » mobilisant deux formes différentes de réflexivité: l'une chez l'artiste et l'autre chez l'observateur. Cette étude s'appuie sur des données ethnographiques recueillies pendant mes enquêtes de terrain doctorales et sur des entretiens avec l'artiste. Après avoir présenté la manière dont les Murui-Muina définissent la danse, leurs bals rituels et la façon dont ce peintre les représente, j'interrogerai la façon dont il se sert de la « condensation rituelle » pour créer son tableau, tout en soulignant le rapport particulier qui relie la toile, le rituel et l'observateur. [Mots-clés : rituel, art, danse, réflexivité, Murui-Muina.]

Representing "with” ritual: reflexivity and ritual dance in a murui-muina painting. Analyzing a painting of the Amazonian painter Brus Rubio Churay dedicated to the murui-muina ritual dances, I will show the particular link that the artist establishes between art and ritual: his painting is less a representation of the ritual and more a representation "with" the ritual. Inspired by the logic of the murui-muina ritual dances, the artist creates a mise en abyme effect mobilizing two different forms of reflexivity: one in the artist and the other in the observer. This study is based on ethnographic data collected during my doctoral fieldwork and on interviews with the artist. After presenting how the Murui-Muina define dance, their ritual balls and how this painter represents them, I will question how the artist uses "ritual condensation" to create his painting, while emphasizing the particular relationship between the canvas, the ritual and the observer. [Key words: ritual, art, dance, reflexivity, Murui-Muina.]

Representando "con” el rito: reflexividad y danza ritual en una pintura murui-muina. Analizando una pintura del pintor amazónico Brus Rubio Churay dedicada a los bailes rituales murui-muina, mostraré el vínculo particular que el artista establece

* Laboratoire d'anthropologie sociale/Centre de recherche et de documentation sur les Amériques [oigarciar@gmail.com]. 
entre el arte y el ritual: su pintura es más una representación “con" el ritual que una representación del ritual. Inspirado en la lógica de las danzas rituales murui-muina, el artista crea un efecto de mise en abyme que moviliza dos formas diferentes de reflexividad: una en el artista y la otra en el observador. Este estudio se basa en los datos etnográficos recogidos durante mis estudios de campo doctorales y en entrevistas con el artista. Después de presentar cómo los Murui-Muina definen la danza, sus bailes rituales y cómo este pintor los representa, analizaré cómo el artista utiliza la "condensación ritual" para crear su pintura, mientras enfatiza la relación particular entre el lienzo, el ritual y el observador. [Palabras clave: ritual, arte, danza, reflexividad, Murui-Muina.]

En regardant Danseuse sur la scène (1876), le célèbre tableau d'Edgar Degas exposé au musée d'Orsay à Paris, nous reconnaissons immédiatement les gestes et les tutus de la danse classique. Mais un observateur n'ayant eu aucun contact préalable avec ce type de représentation serait-il capable de l'identifier sur une toile? Dans la mesure où la réception de l'œuvre dépend de l'expérience culturelle collective à laquelle le spectateur participe (Gombrich et Durand 1987 [1956]; Gombrich et Saxl 2015 [1970]), l'interprétation de telles images dépend de la notion qu' on peut avoir de la danse, qui peut différer grandement de celle de l'artiste. Et que dire de l'analyse des peintures rupestres, par exemple... De semblables défis se posent pour interpréter les œuvres des peintres amérindiens actuels qui représentent des danses rituelles de leurs peuples, dont le moins qu'on puisse dire est qu'elles ne correspondent pas tout à fait à la définition courante du terme « danse ${ }^{1} »$.

La mise en image d'une danse dépend également de la fonction que ses acteurs lui attribuent et de son genre. Des danses différentes - comme par exemple la danse-théâtre ou la danse de salon, la danse contemporaine ou la danse classique - ne vont pas être représentées de la même façon et il en est de même dans les contextes amérindiens qui disposent de nombreuses catégories de danses. Ceci est d'autant plus vrai concernant le genre qui nous occupe ici: la danse rituelle. Les éléments formels, fonctionnels et symboliques ne suffisent pas à identifier une telle danse. Encore faut-il la reconnaître en tant qu'action rituelle. Michael Houseman et Carlo Severi (2009) ont montré qu'une action rituelle se distingue par les relations contradictoires et simultanées qu'elle établit entre les participants. Plus précisément, cette action particulière mettrait en place ce qu'ils appellent une « condensation rituelle » de relations contradictoires. Or, les relations impliquées dans l'action rituelle échappent souvent à la réflexivité des participants. Autrement dit, ces derniers n'ont pas

1. Pour voir des définitions courantes du terme: Judith R. Mackrell, « Dance », Encyclopédie britannique [en ligne], https://www.britannica.com/art/dance, consulté le 20/07/2021. Centre national de ressources texturales et lexicales, « Danse » [en ligne], https://www.cnrtl.fr/ definition/danse, consulté le 20/07/2021. 
Représenter « avec » le rite : réflexivité et danse rituelle dans un tableau murui-muina

toujours la possibilité d'établir « une prise de distance [...] vis-à-vis de soi, de sa pensée, de son action, dans l'intention d'en objectiver les déterminants et les modalités » (Rozenberg 2011, p. 279). De quelle façon ces relations rituelles peuvent-elles alors être figurées? Les tableaux représentant des danses rituelles amazoniennes témoignent-ils d'une « réflexivité rituelle »?

Dans ce travail, j'analyserai une peinture de Brus Rubio Churay - 1'un des artistes murui-muina les plus connus du XXI ${ }^{\mathrm{e}}$ siècle - intitulée La invitación y llegada con fuerza ( $L$ 'invitation et l'arrivée en force, Figure 1, page suivante). Il s'agit d'un tableau appartenant à une série de toiles où l'auteur mobilise un même type de composition ${ }^{2}$. Comment le peintre représente-t-il ces danses et les relations rituelles qu'elles établissent? Que nous dit ce tableau de la « réflexivité rituelle »? Pour tenter de répondre à ces questions, je m'arrêterai sur le sujet peint : les « bals rituels ${ }^{3} »$ murui-muina et leurs participants. Puis je m'intéresserai à la notion de danse chez les Murui-Muina et à la manière dont le peintre représente le « geste dansé ». Enfin j'interrogerai la notion de « condensation rituelle » et la façon dont elle émerge du tableau, ce qui permettra de souligner la relation particulière qui relie la toile, le rituel et l'observateur. Je montrerai que ce tableau est moins une « représentation d'un rituel » qu'une « représentation se servant du rituel ». Par ces analyses, j'établirai que le tableau s'appuie à la fois sur la « réflexivité rituelle » de l'artiste, lui permettant de reconnaître la logique des danses rituelles, et sur celle de l'observateur qui, grâce à un effet de mise en abyme, prend une distance particulière vis-à-vis du rituel.

2. Le sujet et le schème du tableau analysé dans cet article peuvent être aussi retrouvés dans d'autres toiles de Brus Rubio Churay comme par exemple: Pasaporte amazónico 1 (2013); Pasaporte amazónico 2 (2014), https://www.brusrubio.com/2014?lightbox=dataltemiwwimnr43; Invitación al Festival Smithsonian (2015); El mali en el mitayo 1 (2015), https:// www.brusrubio.com/2015?lightbox=dataItem-iwx33ejj7; El mali en el mitayo 2 (2015), https://www.brusrubio.com/2015?lightbox=dataItem-iwx33ejj8; El mali en el mitayo 3 (2015), https://www.brusrubio.com/2015?lightbox=dataItem-iwx33ejj9 et Amanecer al sonido del manguaré (2016), https://www.brusrubio.com/2016?lightbox=dataItem-iwxxu19t5, consultés le 20/07/2021.

3. Ces rituels murui-muina, appelés dans la langue locale rafue et en espagnol bailes (bals), sont des cérémonies collectives dans lesquelles les danses rituelles jouent un rôle fondamental. En espagnol comme en français, le mot «bal » peut être un synonyme de « danse », mais il peut indiquer également des rassemblements festifs concentrés autour des danses. Dans cet article, j'utiliserai le terme «bal » ou «bal rituel » pour désigner ces rassemblements dans lesquels les participants dansent et chantent; tandis que j'emploierai le mot « danse » pour parler des performances chorégraphiques qui caractérisent ces cérémonies. Ce choix permet de différencier les «bals rituels » d'autres genre de « fêtes » organisées par les Indiens amazoniens (comme le carnaval ou d'autres fêtes locales) dans lesquelles la danse ne joue pas le même rôle. 


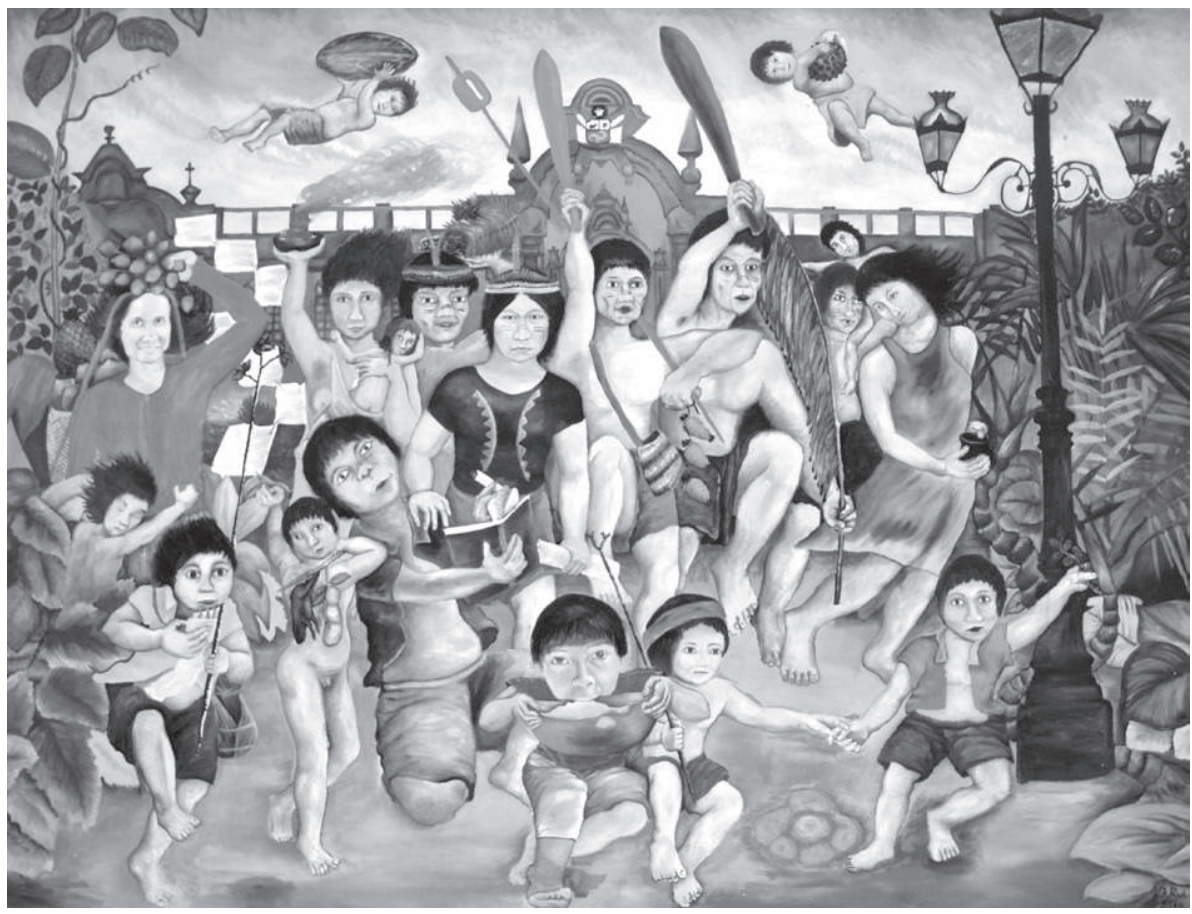

Fig. 1 - Brus Rubio Churay, La invitación y llegada con fuerza (L'invitation et l'arrivée en force), 2014

\section{L'entourage de Brus Rubio Churay : les artistes de Pucaurquillo}

Brus Rubio Churay est issu du peuple Murui-Muina ${ }^{4}$, qui vit dans le NordOuest amazonien, vaste région comprenant toute l'Amazonie colombienne et des parties de l'Amazonie brésilienne, péruvienne et équatorienne. Il est né

4. Les Murui-Muina, un groupe d'environ huit mille personnes, font partie des «Gens du Centre », un ensemble de groupes amérindiens amazoniens qui parlent des langues de différentes familles linguistiques (uitoto, bora, arawak et la langue isolée andoque) et qui considèrent que leur territoire ancestral est la région de Caquetá-Putumayo en Amazonie colombienne et péruvienne. Bien que linguistiquement différenciés, ces groupes partagent une même mythologie et des rituels semblables (parmi lesquels les «bals ») et ils forment ainsi une société régionale unique. Auparavant appelés « Uitoto » (mot signifiant " esclave ») ces Indiens se sont donnés dernièrement le nom « Murui-Muina » en référence aux deux branches dans lesquels sont partagés leurs différents clans. La première branche, appelée « murui », a une connotation masculine et revendique un lien direct avec le Père Créateur Juziñamui. Au contraire, la branche appelée «muina » a une connotation féminine et elle revendique un lien avec Buinaima, le fils de Juziñamui. Les deux branches parlent des dialectes différents de la langue uitoto: les Murui parlent le bue ou le mika alors que les Muina parlent le minika ou le nipode. 
Représenter « avec » le rite : réflexivité et danse rituelle dans un tableau murui-muina

en 1983 au Pérou, dans la région de Pucaurquillo (Loreto) devenue le siège d'un regroupement d'artistes amérindiens dont les travaux s'inspirent des traditions de leurs peuples. Après avoir commencé à peindre dans les années 2000, Brus Rubio Churay a participé depuis 2005 à plusieurs expositions collectives. En 2010, il a organisé sa première exposition indépendante, puis remporté en 2011 le deuxième prix du XIV ${ }^{e}$ Concours national d'Arts visuels « Passeport pour un artiste », organisé par l'Alliance française de Lima. Depuis, il a pris part à plusieurs expositions au Pérou et à l'étranger et il est, à présent, l'un des jeunes artistes amérindiens les plus reconnus d'Amérique latine.

Le travail de Brus Rubio Churay est proche de celui de son oncle, le peintre bora Victor Churay Roque (1972-2002), qui est l'un des fondateurs du groupe d'artistes de Pucaurquillo que certains appellent l' "École de la llanchama » car les artistes peignent sur un tissu de fibres végétales appelé llanchama (Ficus insipida et Brosimum utile; Yllia 2009, p. 95). L'œuvre de Brus Rubio Churay présente également une affinité avec celle d'autres peintres de Pucaurquillo, comme les Murui-Muina Santiago Yahuarcani (né en 1961) et son fils Rember Yahuarcani Lopez (né en 1985).

Brus Rubio Churay et son oncle Victor Churay Roque, à l'instar d'autres peintres de Pucaurquillo, sont engagés dans la lutte pour la protection des droits indigènes. Brus Rubio Churay a par exemple consacré plusieurs tableaux à l'époque de la fièvre du caoutchouc (1879-1912 et 1942-1945), quand les patrons d'entreprises de collecte du caoutchouc ont perpétré à l'encontre des MuruiMuina un génocide qui a fortement transformé l'histoire économique et sociale de toute cette partie de l'Amazonie (Taussig 1991; Pineda-Camacho 2000).

Les rituels sont 1'un des sujets privilégiés des peintres de Pucaurquillo, qui se sont inspirés du courant de l'art visionnaire chamanique et de peintres comme Pablo Amaringo (1938-2009), artiste métis provenant d'une autre région du Nord-Ouest amazonien, celle de Puerto Libertad au Pérou (Luna et Amaringo 1999, Belaunde 2011). Ces artistes peignaient des toiles inspirées des visions chamaniques provoquées par la consommation de plantes hallucinogènes. En revanche, les peintres de Pucaurquillo ne prennent pas de plantes hallucinogènes pour leur processus de création; ils peignent des représentations figuratives des rites plutôt que des visions chamaniques. La invitación y llegada con fuerza de Brus Rubio Churay (Figure 1), que je vais analyser dans les pages qui suivent, est un exemple de ce type de représentation.

\section{Le rituel représenté dans La invitación y llegada con fuerza}

\section{Qu'est-ce qu'un « bal rituel » murui-muina?}

Plusieurs éléments du tableau La invitación y llegada con fuerza, comme certains aliments, l'habillement et les gestes de certains personnages, sont 
caractéristiques des actions rituelles d'un bal, qui sont des célébrations importantes au cours desquelles un très grand nombre d'invités - souvent plus d'une centaine de personnes - dansent sans interruption pendant deux jours ${ }^{5}$. Chez les Murui-Muina, ces célébrations impliquent plusieurs malocas (maisons traditionnelles plurifamiliales) ${ }^{6}$ : les membres de l'une d'entre elles accueillent les chefs et les groupes venus d'une ou de plusieurs autres. Le chef de la maloca invitante, qui dirige le bal et coordonne l'activité rituelle de la cérémonie, est appelé rafue naama tandis que le chef d'une maloca d'invités est appelé fuerama. Dans les rares cas où un bal est organisé par quelqu'un d'autre que le chef de la maloca, c'est ce dernier qui portera le titre de rafue naama.

Le bal rituel ne se limite pas aux deux jours de fête et de danses durant lesquels les invités et les hôtes se rencontrent, mais commence bien avant par plusieurs rituels préparatoires. L'ensemble d'un rituel peut être divisé en cinq parties. Tout d'abord, les hôtes plantent un grand jardin dont la production sera destinée au bal; cette étape est caractérisée par des récitations et des discours rituels nocturnes. Une deuxième période s'ouvre avec la récolte des produits du jardin cérémoniel et avec la préparation de la nourriture pour la fête. La troisième partie du bal rituel est consacrée aux convocations ou invitations de chaque fuerama. C'est à ce moment-là que les invités se rendent à la maloca du rafue naama pour remettre du gibier et ils exécutent alors les premières danses cérémonielles (fakariya $a^{7}$ ). La quatrième partie du rituel constitue le moment central de l'activité cérémonielle: les différents groupes de chanteurs-danseurs, réunis en un seul groupe d'invités, arrivent à la maloca de l'hôte où ils dansent durant deux jours et une nuit. Enfin, pendant la dernière nuit de la fête, a lieu la dernière partie: la clôture du bal. Chacun de ces cinq temps est composé d'un ou de plusieurs rituels. Ce que l'on appelle « bal» n'est donc pas un seul rite mais plutôt une séquence articulée de rituels différents.

\section{Le moment du bal peint par Brus Rubio Churay}

Comme le suggère son titre, le tableau représente la troisième partie d'un bal rituel comprenant deux moments : l'invitation et l'arrivée des invités. Dans un

5. Les bals peuvent avoir lieu à tout moment de l'année, néanmoins les Murui-Muina privilégient les saisons où les fruits sylvestres sont les plus abondants.

6. Les bals ont toujours lieu à l'intérieur de la maloca de l'hôte (rafue naama). Chaque partenaire invité (fuerama) se présente avec son groupe de chanteurs-danseurs. Une fois la fête achevée, l'invité annonce à son tour une célébration à laquelle le chef qui a préparé le premier bal devient l'invité. Les rôles de rafue naama et de fuerama alternent ainsi entre les partenaires rituels. Cette relation perdure leur vie durant.

7. Le terme fakariya dérive du verbe fakade qui veut dire « goûter ». Il se réfère au type de danses réalisées par un seul danseur (accompagné parfois de sa femme) qui a pour fonction de goûter la bière de manioc offerte pour le groupe du maître de bal. Cette danse a lieu la veille des bals. 
Représenter « avec » le rite : réflexivité et danse rituelle dans un tableau murui-muina

premier temps, le rafue naama envoie un messager à la maloca du fuerama, pour l'inviter à la cérémonie et il lui offre un morceau de pâte de tabac, que le fuerama redistribue aux chasseurs de sa maloca. Ces derniers vont alors chasser dans la forêt et ils apportent le gibier au chef hôte, le rafue naama. Au moment de se rapprocher de la maloca du chef hôte, les chasseurs commencent leur chant dansé et, tout en dansant, ils entrent dans la maloca, y déposent le gibier, puis en ressortent. Cette performance constitue le « rituel de remise des gibiers » qui met en lien le fuerama avec le rafue naama au travers du chasseur qui est cependant ici désigné comme zaraima, « danseur » (voir la sous-partie « La performance et l'espace de la danse dans le rite de remise du gibier »).

Dans le tableau de Brus Rubio Churay, on ne trouve toutefois aucune référence à l'invitation. Il y manque aussi l'élément le plus important, la pâte de tabac que le messager - envoyé par le rafue naama - offre au fuerama. En revanche, on peut reconnaître les moments clés du rituel de la remise des produits de la chasse: l'arrivée des invités dansant et le don du gibier. Le tableau représente donc moins « l'invitation » que la réponse à cette invitation, c'est-à-dire l'« arrivée en force » des invités chez le rafue naama.

Nous allons maintenant considérer à la fois le sujet représenté par le tableau, c'est-à-dire le rituel de remise du gibier, et la manière dont le peintre le figure, en présentant, dans un premier temps, les participants au rituel et les personnages du tableau.

\section{Les participants du rite}

\section{Les participants du bal}

Les participants d'un bal rituel murui-muina sont des affins potentiels dans la mesure où le rafue naama et les membres de sa maloca n'ont aucun lien d'alliance matrimoniale avec les malocas invitées. L'affinité potentielle, relation fréquente en Amazonie, présente un degré relativement élevé d'altérité, voire d'inimitié (Viveiros de Castro 2009; Taylor 2009; Fausto et Viveiros de Castro 1993 ; Descola 1993). Un bal est donc un accord entre « ennemis », l'objectif étant de rassembler plusieurs malocas pour faire face à un autre groupe d'ennemis plus puissant, celui des entités non-humaines (animaux ou plantes). Les Murui-Muina, qui considèrent les animaux et les plantes comme des personnes, affirment en effet que les bals rituels sont un moyen de gérer les relations avec ces entités de la nature.

Or, tous les invités sont des affins/ennemis potentiels du rafue naama indépendamment de leur appartenance ethnique. En effet, les invités sont généralement des Murui-Muina ou sont issus d'autres peuples des « Gens du Centre » (l'ensemble de groupes amazoniens auquel les Murui-Muina appartiennent, voir supra, note 4), mais ils se divisent en plusieurs groupes qui « s'identifient » soit 


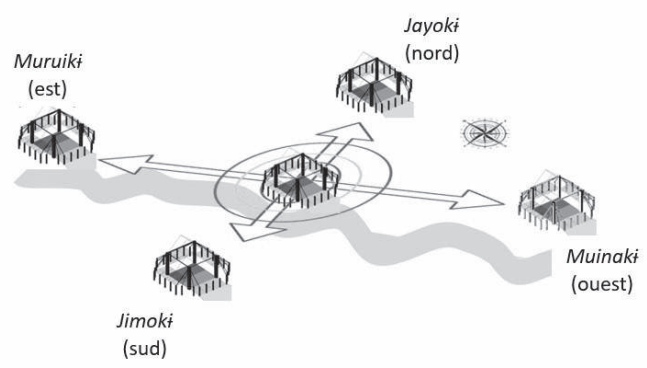

Fig. 2 - Localisation des groupes d'invités par rapport à la maloca invitante (dessin: Garcia 2018)

avec les Murui-Muina eux-mêmes soit avec d'autres peuples qui n'appartiennent pas aux "Gens du Centre ». En général, les invités sont répartis en quatre groupes, ayant chacun un rôle spécifique: les muinaki, les muruiki, les jimoki et les jayoki. Chaque rôle est déterminé par la localisation des habitations des invités par rapport à la maloca de l'hôte. Un chanteur-danseur sera muinama s'il demeure à l'est de la maloca invitante, il sera muruima s'il habite à l'ouest, jimoma s'il réside au sud et jayoma s'il vit au nord (Figure 2). Ces termes sont formés par quatre ethnonymes suivis des suffixes indiquant le singulier $(-m a)$ ou le pluriel $(-k i)^{8}$. Les termes muruiki et muinaki font référence aux deux branches dans lesquelles se divisent les clans murui-muina (les Murui et les Muina); alors que les deux autres groupes représentent des ennemis issus d'autres peuples: les Yagua ${ }^{9}$ et les Nayeni (un groupe aujourd'hui disparu). Les quatre groupes d'invités peuvent être donc classés en deux catégories : ennemis proches et lointains. Les muruiki (ouest) et les muinaki (est) représentent des ennemis proches du rafue naama, appartenant à d'autres lignages mais au même peuple que lui, tandis que les chanteurs-danseurs jimoki (sud) et jayoki (nord) représentent des ennemis plus éloignés, appartenant aux Yagua et aux Nayeni ${ }^{10}$. Autrefois, les différents lignages murui-muina se faisaient régulièrement la guerre entre eux ou avec d'autres groupes des « Gens du Centre » (tous étaient alors considérés comme des ennemis proches); cette guerre était destinée à la chasse de têtes et aux échanges matrimoniaux par le biais du rapt de femmes. En revanche, avec les ennemis lointains, c'est-à-dire avec d'autres Indiens amazoniens n'appartenant pas aux « Gens du Centre », les guerres

8. Le suffixe «-ma» indique un singulier masculin (le singulier féminin est indiqué par le suffixe « - ñ $o$ »), alors que le suffixe « -kit» est employé pour le pluriel (masculin ou féminin).

9. Mes interlocuteurs utilisent le terme «Yagua » pour se référer aux groupes situés au sud du fleuve Putumayo. Néanmoins, les études ethno-historiques montrent que dans cette région, aux Indiens yagua, s'ajoutaient autrefois d'autres groupes de la famille linguistique peba-yagua, désormais regroupés sous le même terme (voir Chaumeil 1994; Goulard 2010).

10. Pour une description plus détaillée des relations entre les invités et le rafue naama, voir Garcia 2016. 
Représenter « avec » le rite : réflexivité et danse rituelle dans un tableau murui-muina

étaient sporadiques et les échanges de femmes ou la chasse aux têtes étaient rares car les Murui-Muina attribuaient à ces groupes des comportements moraux méprisables, semblables à ceux des animaux.

La proximité entre les hôtes et les ennemis proches, interprétés par les invités muruiki et muinaki, explique pourquoi ils sont habillés de façon semblable: ils ne portent pas de costumes particuliers mais seulement leurs vêtements quotidiens (jeans, chemises, T-shirts, etc.). Les muinaki se différencient par l'usage de bouquets de feuilles médicinales tandis que les muruiki sont identifiables par leurs bâtons de rythme. Les bouquets évoquent les vastes connaissances thérapeutiques attribuées au sous-groupe muina (à connotation féminine). Au contraire, les bâtons, représentant des armes, renvoient au caractère guerrier du sous-groupe murui (à connotation masculine). Logiquement, les costumes des danseurs jimoki sont semblables à ceux utilisés par les Yagua (Chaumeil 2000 [1983]) lors de leurs propres rites. Ils sont composés de jupes faites de fibres de palmiers (Attalea butyracea ou Astrocaryum chambira). Les danseurs portent également des coiffes et un bâton de danse ornés de faisceaux de fibres de palmier ${ }^{11}$. Enfin, les costumes du groupe jayoki, renvoyant aux Indiens nayeni, sont caractérisés par des jupes de fibres de chambira (Astrocaryum chambira) qui descendent un peu au-dessous du genou; ils utilisent aussi des branches de palmier. Les bâtons de rythme jimoki et les palmes brandies par les jayoki représentant des armes, évoquant ainsi le rapport d'antagonisme avec ces deux groupes d'ennemis éloignés.

\section{Les personnages du tableau: les participants au rite de remise du gibier}

Trois types de personnes participent de manière directe au rituel qui nous intéresse ici : le rafue naama, les fueraki (pluriel de fuerama) et les chasseurs qui apportent le gibier en dansant, que j'appellerai simplement zaraiki (ou au singulier zaraima $)^{12}$. Chaque zaraima invité appartient donc à l'un des quatre groupes d'ennemis et porte le costume ou les attributs qui lui correspondent. Sur le tableau étudié, nous relevons la présence de dix-neuf personnages, parmi lesquels presque tous ces types peuvent être identifiés.

Dans la partie supérieure, deux enfants s'envolent dans le ciel et portent des fruits de la forêt; Brus Rubio Churay a affirmé, dans une interview ${ }^{13}$, que ce

11. Dans tous les cas, les danseurs peuvent décorer leurs corps et leurs tenues avec des plumes, mais celles-ci ne caractérisent pas un groupe spécifique et peuvent être utilisées par les participants en général (hôtes inclus).

12. Zaraiki est aussi le pluriel du nom féminin zaraiño qui désigne les femmes qui chantent et dansent lors de ces bals rituels.

13. Entretien avec Brus Rubio Churay, 6-7 mars 2019. 
sont des allégories renvoyant à des entités spirituelles de la forêt ${ }^{14}$. Au centre de l'œuvre, nous pouvons voir un homme et une femme portant des couronnes. L'homme a la poitrine peinte en noir, signe caractéristique des chefs muruimuina. Habituellement, lors de ces rituels, le fuerama et les zaraiki arrivent ensemble chez le rafue naama. Étant donné que les danses rituelles ne sont exécutées que par les invités, l'homme portant la couronne de plumes avec la poitrine peinte en noir ne peut qu'être un fuerama et la femme qui se tient à ses côtés ne peut qu'être la cheffe de la maloca invitée (souvent la femme du fuerama). L'artiste représente donc un fuerama accompagné de son épouse et des membres de sa maloca arrivant à la maloca d'un rafue naama.

Autour du fuerama et de sa femme, se trouvent onze personnes, dont trois enfants portés par des femmes et un homme jeune avec un livre ouvert qui représente, comme l'indique le peintre, les étudiants universitaires amérindiens qui se préparent à diriger leurs communautés. Trois autres personnages, deux enfants à la droite de l'étudiant et un homme placé à la gauche du fuerama, portent du gibier attaché à une perche. Quatre femmes sont présentes autour du couple de chefs: une femme non amérindienne portant une chemise violette et un panier de fruits; une femme à la poitrine nue portant un enfant et une calebasse dont s'échappe une fumée de piment, à laquelle est attribué un effet chamanique purifiant; une femme à la poitrine découverte, portant un enfant sur les épaules; et une femme habillée en vert qui appartient à la maloca invitante. Excepté cette dernière, elles portent des offrandes à la manière des femmes invitées qui participent aux rituels de remise du gibier.

\section{Représentation du zaite}

Après avoir identifié le moment du bal représenté sur le tableau et avoir reconnu les participants peints, voyons comment le peintre représente les danses (zaite) et les relations rituelles qu'elles établissent. En répondant à cette question, j'essaie de saisir la manière dont l'artiste exerce une certaine réflexivité lui permettant de reconnaître la logique de construction du rituel (voir Rozenberg 2011; Adell 2016). Pour cela, je définis d'abord la notion de danse propre aux Murui-Muina.

Le zaite et le " geste dansé 》

Chez les peuples amazoniens, les mots utilisés pour se référer à la danse dépassent souvent la signification que nous donnons à ce terme en Occident.

14. Cette représentation apparaît dans d'autres tableaux tels que El casabe y su origen, https://www.brusrubio.com/2011 ?lightbox=dataItem-iwu4sogb2, consulté le 20/07/21. 
Représenter « avec » le rite : réflexivité et danse rituelle dans un tableau murui-muina

D'après le Trésor de la langue française ${ }^{15}$, le mot « danse » indique en premier lieu un «mouvement rythmique du corps ». Il y a toutefois d'autres acceptions qui renvoient de façon différente au mouvement, au rythme ou au corps comme « activité ludique [...] consistant à exécuter une suite de pas », « ensemble de pas, de mouvements déterminés », « série de mouvements élégants, harmonieux ou rythmés », etc. Or la notion amérindienne inclut des éléments supplémentaires parfois inattendus. Le même mot peut indiquer ce que nous appelons « danse » mais aussi d'autres actions, comme l'exécution d'une musique, le chant ainsi que le déguisement ou la consommation de coca ou de tabac.

C'est ainsi que pour les Barasana, peuple amazonien de langue tukano:

Le terme basa renvoie directement aux chants, aux danses, aux instruments de musique, aux ornements corporels (plumes/peinture corporelle); et indirectement à la tenue de certaines rencontres sociales, divers types de discours (enchantements chantés-oratoires, sortilèges de protection), la production de bière de manioc, [la consommation] de coca, de tabac (cigares/tabac), etc. (Stephen Hugh Jones, com. pers., 4 novembre 2015)

De la même façon, chez les Murui-Muina, la notion de danse inclut d'autres actions. Dans la langue murui-muina, le terme rote est utilisé pour nommer, de façon plus générale, l'action de chanter. Le terme jïyirite (" danse ») est utilisé pour désigner les mouvements rythmiques du corps (bras, pieds, tête, etc.). Le terme décrivant le mieux les actions ayant lieu lors des bals murui-muina est cependant zaite, qui désigne l'action simultanée de chanter, de danser et d'arborer des ornements rituels.

Cette performance mobilise plusieurs informations par les paroles des chants, mais aussi par les costumes et les mouvements exécutés qui renvoient à différents types de personnages : les groupes d'ennemis lointains et proches. Le zaite est ainsi un ensemble de chants, de mouvements et de costumes, qui donnent à la performance sa dimension expressive, rappelant que les mouvements de la danse peuvent avoir simultanément des dimensions instrumentales (Mauss 1936, p. 284) et expressives (McNeill, Pedelty et Levy 1990). Tout comme les mouvements ayant une fonction expressive sont appelés gestes, on peut appeler " gestes dansés » les mouvements du corps impliqués dans les danses dont la fonction principale est de fournir de l'information (Fdili Alaoui 2012, p. 43). Dans le cas des actions que décrit le terme zaite, chaque mouvement, indissociable du chant et des costumes, a une signification; chaque mouvement est donc un geste-dansé.

15. Centre national de ressources texturales et lexicales, «Danse » [en ligne], https:// www.cnrtl.fr/definition/danse, consulté le 20/07/2021. 


\section{La performance et l'espace de la danse dans le rite de remise du gibier}

Les rites de remise du gibier sont toujours réalisés par le biais du zaite. Le chasseur portant ses ornements ou des objets caractéristiques s'approche de la maloca du rafue naama en dansant et en chantant. Il poursuit son chemin rituel en continuant à chanter et à danser : il entre dans la maloca où il remet le gibier avant de se diriger vers le rafue naama, pour qui il danse, avant de sortir de la maloca, toujours en dansant, pour rejoindre son groupe à l'extérieur et attendre la suite de la fête (Figure 3).

Tous les chasseurs deviennent chanteurs-danseurs (zaraiki) au moment de livrer le gibier. Le fuerama est le premier à exécuter sa performance. Une fois ce dernier rentré dans la maloca, les autres chanteurs-danseurs démarrent leur zaite d'une façon simultanée mais indépendante. Ce qui pourrait avoir la forme d'un seul rituel collectif est plutôt un ensemble de plusieurs petits rituels simultanés de remise du gibier. La performance de ces zaraiki permet de délimiter les zones constituant l'espace de la danse. Ces zones ainsi que le passage de l'une à l'autre permettent de mieux situer la scène représentée par le peintre. Le chanteur-danseur (zaraima) débute son chant dans le patio devant la maloca (Figure 4: zone A). Cette première zone inclut aussi l'espace entre la porte d'entrée et les deux premiers poteaux de l'habitation. Ensuite, l'action se déroule au centre de la maloca, dans le carré compris entre les quatre poteaux principaux. Ce carré, qui est le véritable espace cérémoniel, se divise en deux parties, qui sont respectivement la deuxième et la troisième zone de la performance. Dans la deuxième zone (Figure 4 : zone B), chaque chanteurdanseur remet le gibier au chef de la maloca. Les hôtes viennent le récupérer discrètement sans interrompre le chant dansé du zaraima. La troisième zone (Figure 4: zone C) commence à l'endroit où les femmes de la maloca invitante rencontrent les zaraiki et se prolonge jusqu'au jíbibirit ${ }^{16}$, partie de la maloca

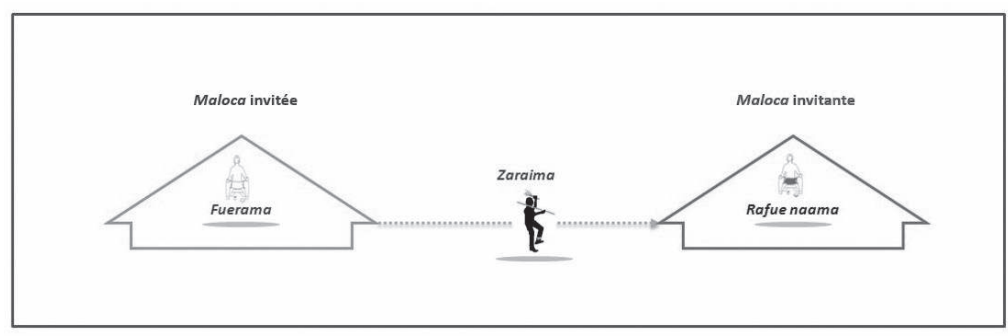

Fig. 3 - Schème général du rituel de remise du gibier (dessin: Garcia)

16. Il s'agit de l'endroit destiné à la préparation et à la consommation rituelles des feuilles de coca et de tabac. Cet espace est délimité par plusieurs bancs (raiirabiki) dont le plus important est celui du chef. Il n'y a ni table ni aucun autre artefact. 
Représenter « avec » le rite : réflexivité et danse rituelle dans un tableau murui-muina

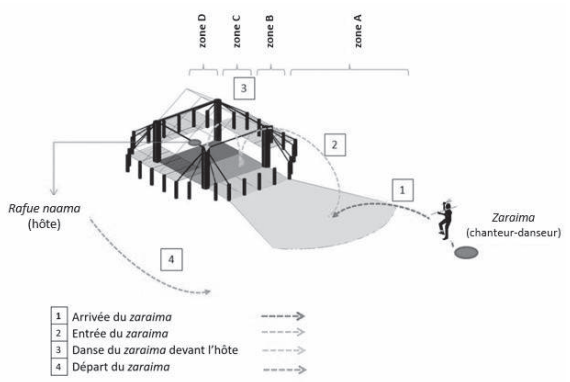

Fig. 4 - Schéma des déplacements du danseur (zaraima) dans une maloca lors du rituel de remise du gibier (dessin: Garcia)

située à l'opposé de la porte d'entrée et où tous les participants reçoivent de la coca et du tabac (il s'agit de l'espace nommé mambeadero dans l'espagnol local) (Figure 4 : zone D).

Le climax de cette performance a lieu au centre de la maloca (entre les zones $\mathrm{B}$ et $\mathrm{C}$ ). Arrivé à ce point (zone $\mathrm{B}$ ), le zaraima est stoppé par les femmes de la maloca invitante qui lui proposent une calebasse remplie d'un jus de manioc doux (manicuera en espagnol ${ }^{17}$ ) qu'il doit boire avant de rejoindre le rafue naama. Cette boisson est censée adoucir le zaraima et le rendre inoffensif. Le zaraima, quant à lui, cherche à rejoindre le rafue naama avec toute sa charge agressive, ce qui l'amène à essayer de franchir le barrage féminin en créant une dynamique comique: plus il essaie de contourner les femmes, plus elles vont insister en lui offrant de la boisson. Pendant cette partie de la performance, le zaraima n'arrête jamais son zaite. La présence des femmes détermine cependant un changement dans le rythme de la danse. S'installe, en effet, un jeu de séduction qui est central dans le bal rituel. Les invités et les hôtes, étant des affins potentiels, lors de ce rituel, sont censés figurer des rapports de guerre et de mariage. Quand les femmes parviennent finalement à le bloquer, le zaraima prend la calebasse tandis qu'elles lui mettent sur les épaules des galettes de manioc et des tamales $^{18}$ (voir Figure 4). Il peut ensuite rejoindre le rafue naama tout en restant dans le carré délimité par les quatre poteaux principaux (Figure 4 : zone $C$ ) et sans rentrer dans la dernière zone, celle du mambeadero (Figure 4 : zone D).

17. La manicuera (Manihot esculenta) est une variété de manioc particulièrement sucrée mais vénéneuse. Elle est consommée sous forme de boisson (juiñoi): on râpe les tubercules mûrs; la pâte ainsi obtenue est pressée dans une passoire pour séparer le jus de la fibre, on la laisse décanter pour séparer les amidons et enfin on la fait cuire pour éliminer le poison. Le juiñoi est une boisson non fermentée. Comme je l'ai montré dans ma thèse (Garcia 2018), le manioc et, plus particulièrement, la manicuera sont associés au monde féminin. Dans le contexte murui-muina, cette plante a la capacité d'enlever les intentions négatives.

18. Terme espagnol désignant un pain fait à base de manioc cuit. 
Chacun des quatre groupes d'invités - muruiki, muinaki, jayoki et jimoki (voir Figure 4) - possède des pas de danse différents. Ils peuvent être distingués à partir du système élaboré par le théoricien de la danse Rudolf Laban (1879-1958) qui décrit le mouvement en fonction des axes directionnels, des orientations, de la position du corps et du changement de son centre de gravité (Laban 1994). Les muinaki dansent avec des pas relativement courts alternant des mouvements d'avant en arrière, tout en pivotant fréquemment. Ils tiennent des bouquets de feuilles dans une ou dans les deux mains, qu'ils agitent vers l'extérieur. En revanche, les muruiki se meuvent toujours avec de légers déplacements latéraux. Ils accentuent leurs pas en marquant le rythme avec un bâton qu'ils frappent sur le sol. Ils suivent en général un axe parallèle à la ligne imaginaire entre les portes antérieure et postérieure de la maloca, soit de l'est vers l'ouest (Garcia 2016). Les danseurs jimoki et jayoki se déplacent de façon similaire, avec des pas latéraux, normaux ou longs, et des mouvements lents et bas. Les jimoki se distinguent notamment par leurs bâtons de rythme qu'ils font pivoter et qu'ils pointent, dans certains cas, contre les hôtes en imitant un geste d'agression. Pour sa part, le trait le plus caractéristique des jayoki sont les branches de palmier de deux à trois mètres de long avec lesquelles ils dansent tout au long de la cérémonie.

Après avoir défini l'espace du rituel et les mouvements caractéristiques des danses, voyons à présent de quelle manière Brus Rubio Churay a représenté le zaite lors du rituel de remise du gibier et quels gestes il a retenus.

\section{La représentation $d u$ zaite}

On peut distinguer dans le tableau sept chanteurs-danseurs zaraiki. Dans la partie intermédiaire, deux sont placés à la gauche du chef, soulevant chacun un bâton avec le bras droit. L'un tient dans la main gauche une branche de palmier qui permet de l'identifier au groupe des jayoki, soit les invités habitant au nord de la maloca où a lieu le bal et représentant des ennemis éloignés. Le deuxième a dans sa main gauche une perche rouge d'où pendent des larves comestibles (Rhynchophorus palmarum), qui sont considérées comme des gibiers. Puisque ce dernier ne porte ni le costume particulier des jayoki ou des jimoki, ni le bouquet de fougères des muinaki, il ne peut qu'appartenir aux muruiki, les invités situés à l'ouest de la maloca qui représentent la branche murui des Murui-Muina. Finalement, ces éléments ne sont pas de simples costumes mais une partie signifiante du zaite, qui indique l'identité des danseurs. Ils se retrouvent toujours dans les toiles que Brus Rubio Churay a consacrées aux danses rituelles, avec parfois quelques variations. Le peintre représente à chaque fois un ou plusieurs éléments caractéristiques des costumes : les masques, le maquillage, les couronnes en plumes, les grelots (des bracelets et des chevillières de graines qui sonnent et marquent le rythme), les bouquets, les bâtons de rythme et les perches ou les paniers contenant des offrandes. 
Représenter « avec » le rite : réflexivité et danse rituelle dans un tableau murui-muina

Les deux personnages à la gauche du chef ont le bras droit levé, l'un des deux genoux pliés au niveau de la poitrine et l'autre jambe posée sur le sol, légèrement fléchie, leitmotiv que l'on retrouve dans d'autres peintures de Brus Rubio Churay. Par exemple, dans le tableau Fakarilla (El saludo) ${ }^{19}$, où est représenté un groupe de zaraiki se préparant à boire de la bière de manioc $\left(\right.$ cahuana $\left.^{20}\right)$, on peut voir ce même mouvement représenté de profil. Deux personnages, l'un masqué ${ }^{21}$ et l'autre portant une couronne de plumes ${ }^{22}$, avancent avec la jambe gauche posée au sol et légèrement pliée et le genou droit également plié, les bras en l'air et le corps en position diagonale. De cette façon, le peintre représente le mouvement. Mais comment l'observateur peut-il conclure qu'ils sont en train de danser? Pour préciser qu'il s'agit d'un geste dansé, Brus Rubio Churay a inséré les costumes caractéristiques et des éléments renvoyant au rythme de la danse. En effet, les pieds des personnages sont ornés avec l'un des rares instruments qui accompagnent les danses murui-muina, les grelots. Dans d'autres tableaux, comme il le fait dans La invitación y llegada con fuerza, l'artiste représente aussi les bâtons de rythme ${ }^{23}$. Ces éléments nous renseignent également sur ce qu'exprime le geste dansé. En fait, les bâtons de rythme ainsi que les perches portant le gibier, les deux employés lors des danses, sont une évocation des relations belliqueuses entre humains et non-humains que ces performances font revivre ${ }^{24}$.

En revenant à La invitación y llegada con fuerza, on voit à la gauche des danseurs une femme courbée portant une calebasse. Ce mouvement pourrait être

19. Voir: Brus Rubio Churay, Fakarilla (El saludo) (Fakarilla [La salutation]), 2014, https://www.brusrubio.com/2014?lightbox=dataItem-iwwimnr31, consulté le 20/07/21.

20. La cahuana est l'une des boissons utilisées dans les bals murui-muina. Elle est faite à base d'amidon de manioc et est adoucie avec des jus de divers fruits. Elle est parfois appelée « bière de manioc » même s'il ne s'agit pas d'une boisson fermentée. La cahuana est utilisée en tant que rétribution des dons apportés par les invités du bal.

21. Certains bals rituels murui-muina exigent l'usage de masques. Pourtant, le masque figuré dans ce tableau correspond à un type de rituel célébré par les Bora. Comme je l'ai expliqué plus haut, les « Gens du Centre » (Bora, Miraña, Okaina, Muinane, Murui-Muina, etc.) partagent une bonne partie de leur mythologie et de leurs rituels et il n'est donc pas rare que des danseurs de ces différents peuples participent à un même bal. Brus Rubio Churay m'a en effet confirmé que, dans ce tableau, le danseur masqué représente un danseur bora (Rubio Churay, conversation personnelle, mars 2019).

22. La couronne est un élément distinctif des chefs murui-muina. Mais ces ornements sont parfois utilisés pour exprimer l'origine amérindienne des sujets du tableau.

23. Voir: Brus Rubio Churay, Fakarilla: fiesta de pedido de fruta (Fakarilla: fête pour demander des fruits), 2011, https://www.brusrubio.com/2011?lightbox=dataItem-iwu7mnec, consulté le 20/07/21. Voir: Brus Rubio Churay, Fakarilla (Saludo) (Fakarilla [Salutation]), 2009, https:/www.brusrubio.com/2009?lightbox=dataItem-iwu1wzii2, consulté le 20/07/21.

24. Chasse et guerre se présentent en effet comme des activités analogues et les animaux abattus sont traités comme des victimes de guerre puisqu'ils ont un statut équivalent à celui des humains pour les Murui-Muina (sur ce point, voir Garcia 2018). 
interprété comme une danse. On n'y voit cependant ni les éléments caractéristiques du zaite, ni le leitmotiv employé par Brus Rubio Churay pour l'évoquer: la femme n'est pas en train de taper le sol avec le pied comme le ferait un zaraima et elle ne porte pas non plus d'offrandes comme le font les femmes invitées. Il s'agit en fait d'une femme de la maloca invitante offrant du jus de manioc aux invités. En revanche, aux extrémités du tableau (à droite et à gauche) on peut distinguer quatre danseurs. Trois sont représentés avec le leitmotiv indiquant la danse: l'enfant qui joue de la flûte de pan et les deux enfants se tenant la main ont tous les trois un genou plié et une jambe au sol à la manière des deux zaraikì (mais ils ne lèvent pas leurs bras). L'autre enfant, nu, est le seul danseur à ne pas être représenté avec un genou plié. Il lève cependant un bras et porte du gibier, ce qui laisse entendre qu'il pourrait bien être en train de danser.

Nous pouvons déjà identifier dans le tableau deux des trois éléments constituant le zaite: la danse et les tenues. Le troisième élément, le chant, ne semble pas y figurer. Brus Rubio Churay représente souvent les visages de façon plutôt neutre, presque inexpressive. En outre, alors que son oncle, Victor Churay Roque, enrichit souvent ses tableaux avec des vignettes et des textes ${ }^{25}$, Brus Rubio Churay ne reporte pas les énonciations verbales chantées, pas plus qu'il ne semble les évoquer de façon symbolique. Pourtant, on observe sur La invitación y llegada con fuerza que certains chanteurs-danseurs ont la bouche légèrement entrouverte. L'enfant à la chemise jaune (dans le registre bas) semble souffler dans sa flûte. L'enfant à la chemise rouge ainsi que les deux zaraiki ont la bouche également entrouverte, bien qu'ils ne soient pas en train de jouer d'un instrument à vent. Il est possible que le peintre ait voulu ainsi représenter l'action de chanter. Quoi qu'il en soit, il faut considérer le chant comme sous-entendu dans la représentation du zaite, étant donné que ce terme renvoie à une action unique dans laquelle le chant (rote) et la danse (jiyirite) sont indissociables.

\section{La « condensation rituelle » et les enjeux de la réflexivité}

Une fois analysée la représentation de cette performance particulière qu'est le zaite, nous pouvons interroger, d'une part, la façon dont le peintre a rendu compte du caractère rituel d'une telle danse et, d'autre part, les formes de réflexivité que ce tableau implique. Pour ce faire, il faut revenir sur la notion de «condensation rituelle » et sur la façon dont elle se réalise dans le rite ici représenté.

25. Voir par exemple: Victor Churay Roque, Los castigos a los boras (Les châtiments des Indiens bora), 2001 (Cortés Garzón 2015, p. 105). 
Représenter « avec » le rite : réflexivité et danse rituelle dans un tableau murui-muina

\section{«La condensation rituelle » lors du rite de remise de gibier}

Lorsqu'ils viennent livrer les gibiers au rafue naama, les zaraiki ne sont pas seulement en train de danser et de chanter, ils participent à un rituel. Pour les Murui-Muina, ces danses sont en effet des $«$ danses rituelles $»^{26}$. Or Michael Houseman et Carlo Severi définissent les rituels à partir de la « condensation rituelle » : une action est un « rite » si elle produit une conjonction de relations simultanées et contradictoires entre deux ou plusieurs participants. Une telle « condensation rituelle » se vérifie dans tout rite et permet de le distinguer d'autres événements (Houseman et Severi 2009). Si le zaite est une danse rituelle, de quelle façon contribue-t-il à la « condensation rituelle » qui se réalise lors du rite de remise des gibiers? Quelle est la relation « simultanée et contradictoire » mise en place entre les participants? Jusqu'à quel point le peintre rend-il compte d'une telle condensation dans son œuvre?

Les significations du zaite, par les mouvements, les chants et les costumes, ne se limitent pas à "représenter » les caractéristiques d'ennemis proches ou éloignés. La fonction du zaite n'est pas non plus de la figuration ou de la mimesis. Dans le zaite, comme dans tous les rituels et dans l'ensemble du rite de remise du gibier, les participants se comportent comme s'ils étaient d'autres entités et ils sont traités comme s'ils étaient ces entités. Ils ne jouent pas un rôle mais deviennent d'autres êtres, ils subissent une « identification », selon le terme employé par Bateson (1990 [1936], p. 63). Durant le bal murui-muina, les zaraiki venant de la maloca du fuerama placée au nord de la maloca du rafue naama, s'identifient toujours aux Indiens nayeni. Ceux venant du sud acquièrent l'identité des Indiens yagua. Les chanteurs-danseurs venant de l'est s'identifient à la branche muina et ceux venant de l'ouest à la branche murui (Figure 2). Cette identification des zaraiki avec des groupes d'ennemis proches ou lointains est créée par le zaite: par les costumes, les bâtons de rythme ou les feuilles de palmier, par les chants et par les mouvements. La réflexivité rituelle des danseurs est relative: les zaraiki peuvent avoir une prise de distance vis-àvis de leurs actions mais ils ignorent souvent le rapport d'antagonisme que ces actions établissent à l'égard du rafue naama. En revanche, ils sont davantage conscients de l'identification mobilisée par les mots chantés. En effet, lorsqu'il chante (et danse), le zaraima s'adresse fréquemment au rafue naama en usant d'un terme de parenté - moo (père) (Garcia 2018). Il emploie également d'autres termes destinés aux consanguins tels que eiño (mère), aama (frère) ou miriño (sœur ${ }^{27}$ pour s'adresser aux autres hôtes. Le chant permet ainsi au zaraima de

26. Pour une analyse plus détaillée de ce point, voir Garcia (2018).

27. Selon la terminologie de la parenté murui-muina, aama (frère) et miriño (sœur) sont des termes utilisés pour un Ego masculin. Un Ego féminin utilisera plutôt les termes ï̈ioi (frère) et evuño (sœur) respectivement. 
s'identifier à un membre du groupe de filiation du rafue naama ${ }^{28}$. Le zaraima, devient alors un affin potentiel (ou ennemi) du rafue naama, grâce à la danse et aux tenues; en même temps, il devient un membre de son groupe de filiation grâce au chant dans lequel il utilise des termes qui dénotent un lien de parenté proche avec le rafue naama. Le zaite est donc une action permettant d'établir deux relations simultanées et contradictoires, parfait exemple de ce que Houseman et Severi appellent la « condensation rituelle ». Les participants témoignent de différents niveaux de réflexivité rituelle concernant ces relations.

\section{«La condensation rituelle » et le tableau: représenter « avec » le rituel}

Dans le tableau La invitación y llegada con fuerza, les deux zaraiki qui se trouvent à la gauche du fuerama sont reconnaissables à leurs costumes et à leurs attributs: celui qui porte une branche de palmier est un invité venant du nord (jayoki) qui s'identifie aux Indiens nayeni; l'autre, celui qui porte une perche avec des larves est un muruikit, un invité venant de l'ouest s'identifiant à la branche murui. Les deux dansent en soulevant des bâtons de leur bras droit (le peintre affirme qu'il s'agit du type de bâton qui était utilisé durant les guerres tribales). La femme habillée en vert vient leur offrir du jus de manioc dans une calebasse qui, pour les Murui-Muina, purifie les invités de leur agressivité; cette femme appartient donc au groupe des hôtes. Ces éléments représentent donc également l'antagonisme entre les invités et les hôtes. Cependant, aucun élément du tableau ne renvoie explicitement à l'autre relation, celle de filiation entre les invités et le rafue naama. En fait, comme nous l'avons souligné, les paroles des chants qui expriment cette relation ne sont pas directement figurées et le chant n'est évoqué que par des bouches entrouvertes.

Les indices de deux relations contradictoires et simultanées apparaissent pourtant si on considère certaines références du tableau avec des personnes et des lieux particuliers. Dans le tableau, on peut voir plusieurs personnes de l'entourage de Brus Rubio Churay. Le peintre explique que la femme à la

28. Cette identification mobilisée par les invités correspond à celle des hôtes lors des rites précédant le rituel de remise du gibier. Lors de la deuxième partie du bal, le rafue naama réalise en effet un ensemble de rituels destinés à reconstituer chaque espace-temps de la Création (Garcia 2019). L'objectif est de mobiliser une identification progressive entre l'hôte et le Créateur du monde. Une telle identification autorise l'hôte à se comporter comme s'il avait réalisé lui-même les actions du Créateur et autorise aussi les participants du rituel à le traiter comme s'il avait vraiment réalisé de telles actions créatrices. Ce changement a un effet sur l'identité de tous les participants du bal qui désormais se comportent comme des membres du groupe de filiation de l'hôte: les participants au bal bénéficient alors de la protection du rafue naama. Lors du rituel, l'identification réalisée par l'hôte transforme temporairement les relations d'affinité (celles qui, en Amazonie, sont qualifiées comme des relations de prédation) - qui gouvernent par définition les rapports entre la maloca invitante et les autres malocas participant au bal - en relations de filiation (Garcia 2018). 
Représenter « avec » le rite : réflexivité et danse rituelle dans un tableau murui-muina

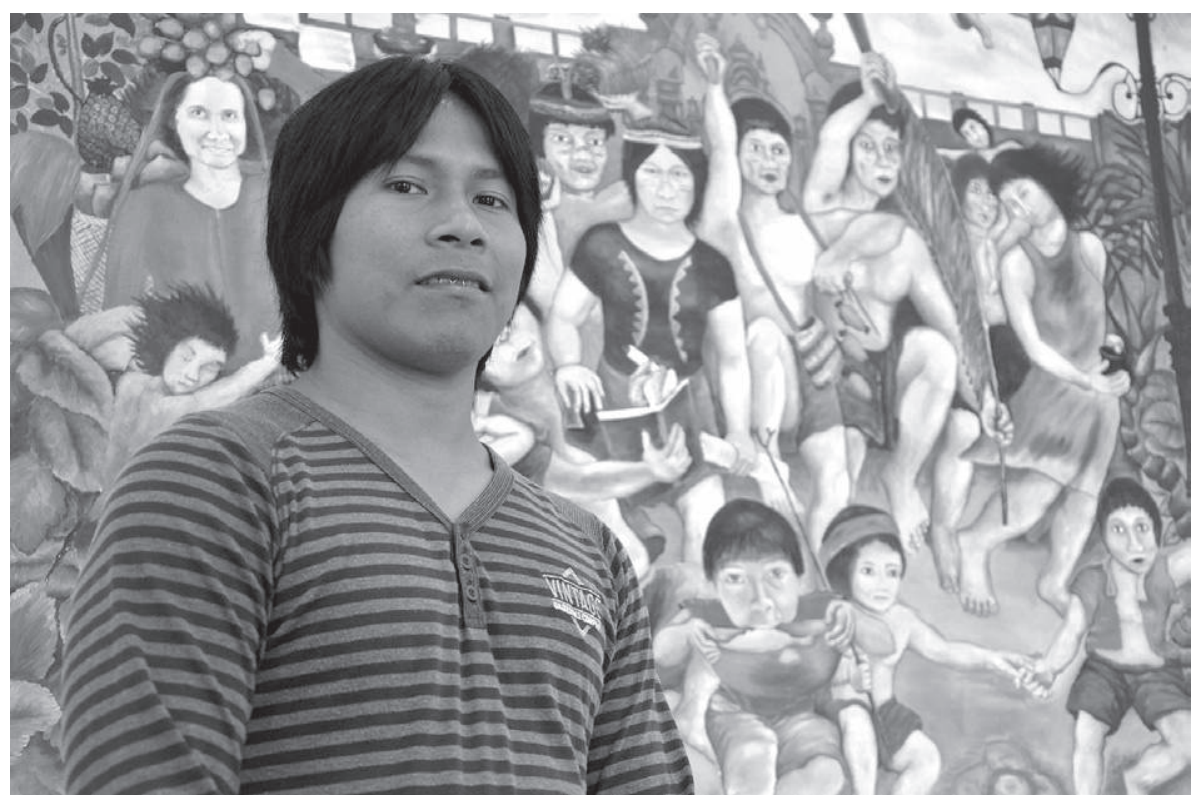

Fig. 5 - Brus Rubio Churay devant son tableau La invitación y llegada con fuerza (photo: M. R. Colombie, 2015)

chemise violette a les traits d'une amie non-indienne; que l'enfant avec la calebasse est l'un de ses neveux et que le chef indien au centre est son autoportrait (Figures 1 et 5$)^{29}$. Le peintre spécifie donc l'identité de certains personnages agissant en tant que zaraiki et il en va de même pour les lieux qui sont censés évoquer la maloca du rafue naama et celle du fuerama.

Pour évoquer la maloca du fuerama, le peintre introduit des éléments renvoyant au chef invité et à son lieu d'origine. Brus Rubio Churay affirme que l'ensemble des danseurs viennent de la forêt et que c'est la raison pour laquelle il a dépeint la scène entourée d'une végétation sylvestre et d'un ciel bleu. Il ajoute qu'il a peint le sol de couleur jaune pour évoquer le sol à l'intérieur des malocas murui-muina qui est toujours en terre. La scène semble ainsi située dans une maloca murui-muina. Mais le rituel de remise des produits de la chasse n'est-il pas censé avoir lieu dans la maloca du rafue naama? En réalité le peintre situe cette scène dans deux endroits qui se superposent (Figure 6). Sur le tableau nous pouvons en effet voir la Plaza Mayor de $\operatorname{Lima}^{30}$, qui est évoquée par deux

29. Entretien avec Brus Rubio Churay, 6-7 mars 2019.

30. Cette place rassemble plusieurs bâtiments emblématiques d'institutions importantes pour la nation péruvienne: la cathédrale de Lima, l'église du Sagrario, le palais de l'Archevêque, le palais municipal et enfin le palais présidentiel de l'État péruvien. 


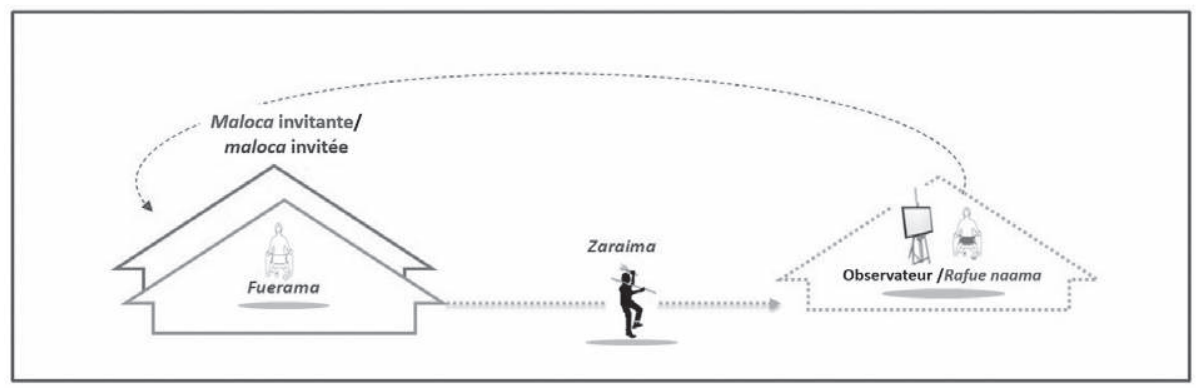

Fig. 6 - Schème de remise des gibiers dans La invitación y llegada con fuerza, Brus Rubio Churay (dessin: Garcia)

images. À l'arrière-plan, on peut reconnaître le bâtiment le plus représentatif de cette place: le palais présidentiel de l'État péruvien ou Casa de Pizarro (Figure 7). Au premier plan, on trouve une lanterne vénitienne à trois bras, typique de la Plaza Mayor. La scène se situe donc en même temps dans la forêt amazonienne et à Lima. Pour le peintre, la forêt évoque la maloca du fuerama, tandis que la Plaza Mayor correspond à la maloca du rafue naama.

D'ailleurs, où se trouve le rafue naama? On aura déjà remarqué que le peintre a représenté tous les participants au rituel de la remise des produits de la chasse, à l'exception du principal, le chef de la maloca invitante, c'est-à-dire le maître du bal, le rafue naama. Brus Rubio Churay affirme que le rafue naama est le président du Pérou, qui réside dans la Casa de Pizarro (Figure 7) ${ }^{31}$. Ceci explique également qu'il a choisi de situer la scène sur la Plaza Mayor de Lima et qu'il identifie le groupe du rafue naama à la société péruvienne ${ }^{32}$. Mais où se trouve le président du Pérou au moment où le fuerama Brus Rubio Churay et ses amis zaraiki viennent lui offrir le gibier? Serait-il enfermé dans son palais? Des indices fournis dans le tableau peuvent nous indiquer sa position.

Brus Rubio Churay participe souvent aux bals rituels en tant que zaraima mais il n'a pas encore suivi la formation chamanique pour devenir rafue naama ou maître de bal. Cela ne l'a pas empêché pour autant de composer le tableau selon le schème de cette cérémonie. En retenant cette partie du rituel, il ne fait aucun doute que, pour lui, il s'agit d'un moment du bal ${ }^{33}$. Lors du rituel de remise

31. Entretien avec Brus Rubio Churay, 6-7 mars 2019.

32. Dans d'autres tableaux, la position de rafue naama semble être attribuée aux autorités d'autres États comme dans les tableaux Pasaporte amazonico 2 (2014) et Invitacion al Festival Smithsonian (2015) où cette place est assignée respectivement aux chefs d'État français et étasunien.

33. Tout comme les participants du rituel, les artistes le représentant ne possèdent souvent que des visions " incomplètes et contradictoires » sur la signification de leurs actions (Humphrey et Laidlaw 2004, p. 80). 
Représenter « avec » le rite : réflexivité et danse rituelle dans un tableau murui-muina

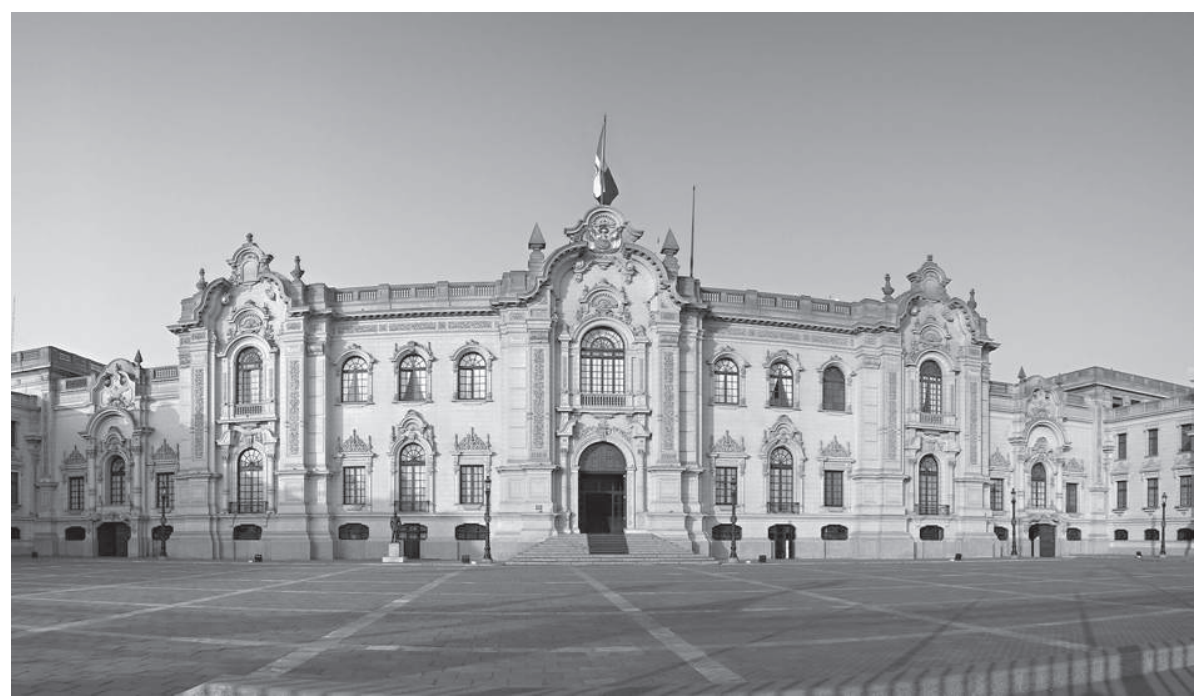

Fig. 7 - Palais présidentiel de l'État péruvien ou Casa de Pizarro (Maison de Pizarro) (photo : Felipe Forte, 2007, https://www.flickr. com/photos/fortes/2090238532/, consulté le 20/07/21)

du gibier, les zaraiki suivent un axe de déplacement idéel reliant leur maloca à la maloca du rafue naama. Or, bien que dans le tableau les deux malocas se superposent, on peut percevoir la direction du déplacement des danseurs. Plusieurs éléments indiquent que les personnages se dirigent vers la maloca du rafue naama plutôt que vers celle du fuerama. Puisqu'ils ont le gibier à la main, l'offrande n'a pas encore été faite au rafue naama. De surcroît, l'offrande de la boisson de manioc (cahuana et manicuera) n'a pas encore eu lieu. On le comprend parce que la femme à la robe verte vient pour offrir du jus de manioc aux danseurs, mais également parce que l'enfant au centre (registre inférieur) est, selon Brus Rubio Churay, en train de recevoir une calebasse remplie de bière de manioc (cahuana). Étant donné que ce sont toujours les hôtes qui offrent ces boissons aux invités lors du rituel, nous pouvons en conclure que le groupe se trouve dans la maloca du rafue naama et qu'il se déplace en direction du chef hôte (Figure 3), auquel les chanteurs-danseurs sont censés s'adresser.

Brus Rubio Churay (le fuerama-peintre), son « amie » non-indienne et son neveu regardent dans la même direction, qui coïncide avec la position de l'observateur. De cette manière, l'artiste introduit l'observateur du tableau dans sa composition: il crée un effet de mise en abyme qui augmente le niveau de réflexivité de l'observateur (voir Bal 1978; Kintsurachvili 2010). Cet effet ressemble à celui décrit par Foucault dans l'interprétation qu'il livre du tableau le plus célèbre du Siècle d'or espagnol (1492-1681): Les Ménines (1656-1657) 
de Velázquez ${ }^{34}$. Dans Les Ménines, on voit ce dernier en train de peindre une toile représentant la cour de Philippe IV; à côté de l'infante Marguerite-Thérèse, se tiennent des demoiselles d'honneur, une naine et d'autres personnages encore. L'artiste se penche sur le côté de sa toile, dont on ne perçoit que le revers, et il regarde devant lui, comme d'autres personnages, vers l'endroit où se trouve son modèle. Il est alors l'observateur du tableau:

Au moment où ils placent le spectateur dans le champ de leur regard, les yeux du peintre le saisissent, le contraignent à entrer dans le tableau, lui assignent un lieu à la fois privilégié et obligatoire, prélèvent sur lui sa lumineuse et visible espèce, et la projettent sur la surface inaccessible de la toile retournée. (Foucault 1966, p. 21)

Si la démarche d'inclusion est similaire, Brus Rubio Churay se place par contre au centre de son œuvre La invitación y llegada con fuerza et son regard de fuerama-peintre se pose alors en miroir inversé de l'observateur extérieur que nous sommes. Foucault ajoute que la place du spectateur du tableau, coïncidant avec la place du modèle est en réalité la place du roi. Dans Les Ménines, Velázquez se serait en fait représenté au moment de réaliser un portrait du roi Philippe IV et de son épouse. Le spectateur de La invitación y llegada con fuerza se trouve également « à la place du roi », qui en l'occurrence est la place du rafue naama ou du président du Pérou (Figure 6). Cette démarche n'est en rien égotiste, mais elle porte un message dont le peintre est conscient, son œuvre sera vue par ce qu'il dénonce, l'extérieur, toute personne qui pourra voir cette réalisation. Il se positionne en tant qu'observateur-observé.

Le tableau est de prime abord un dispositif qui projette l'observateur dans l'action rituelle, mais pas seulement. C'est en introduisant l'observateur dans le dispositif de la toile que le peintre parvient à « reconstituer» l'action rituelle. Une fois reconnue la structure du tableau, il est donc possible de se situer dans l'un des groupes du schème fuerama/zaraima/rafue naama, de se voir intégré dans le groupe du rafue naama et de se reconnaître dans la relation d'opposition, en même temps que de fraternité, à l'égard des zaraiki et du fuerama. Créer simultanément des sentiments d'antagonisme et de protection chez l'observateur est l'un des deux objectifs de l'artiste.

La invitación y llegada con fuerza montre la façon d'arriver dans une fête [bal rituel], en toute fraternité, avec toutes ces énergies, avec tous ces instruments mythologiques ou chants mythologiques [...]. Mais en même temps, je veux

34. Brus Rubio Churay reconnaît l'influence d'autres peintres européens sur ses toiles. Il affirme par exemple que son tableau Comunicacion dicotomizada (Communication dichotomisée) révèle l'influence du dadaïsme sur son travail ou encore que son œuvre Las hijas del curaca (Les filles du chef) est inspiré du tableau Les Trois Grâces (1639) de Rubens. Il ne serait donc pas surprenant qu'il soit aussi influencé par un artiste comme Velázquez. 
Représenter « avec » le rite : réflexivité et danse rituelle dans un tableau murui-muina

représenter ici comment... [la toile] est une façon de protester aussi... de protester avec la fête, avec des chants, en toute connaissance ${ }^{35}$.

Cela explique pourquoi l'artiste dépeint le groupe d'invités indiens exécutant des pratiques chamaniques telles que l'usage de piments fumés ${ }^{36}$ destinées à provoquer des effets bienfaisants aussi bien que des actions belliqueuses comme celles représentées par les bâtons des zaraikit ${ }^{37}$. Ces attitudes opposées sont également évoquées par deux autres objets. Brus Rubio Churay affirme que les documents que le chef tient à la main sont les demandes et les réclamations adressées aux ONG et à l'État péruvien par les communautés amérindiennes, tandis que le livre tenu par le personnage agenouillé représente les connaissances des jeunes universitaires indiens « acquises » de la société péruvienne. En exerçant un type de réflexivité lui permettant de déchiffrer la logique de construction du rite (réflexivité épistémique; Rozenberg 2011), l'artiste dépeint donc des actions renvoyant à une relation d'opposition entre le monde indigène et la société péruvienne. Il montre ainsi les relations d'antagonisme et de protection propres à la condensation rituelle. Ces relations, simultanées et contradictoires, s'actualisent à chaque fois qu'un observateur se place devant le tableau. La composition du tableau, donnant lieu à cette mise en abyme, permet donc d'augmenter le niveau de réflexivité de l'observateur envers le rituel en ce qu'il passe d'une attitude passive à une autre active devant un tableau qui est construit en se servant de la logique du rite.

\section{Conclusion}

L'analyse de La invitación y llegada con fuerza offre un aperçu du vaste système qui peut inspirer un peintre amazonien comme Brus Rubio Churay. À la façon d'un horloger qui s'intéresse au mécanisme d'une montre et pas seulement à la beauté du cadran, nous avons essayé de comprendre cette œuvre d'un peintre en interrogeant ses engrenages.

35. Version originale: "La llegada con fuerza [sic] es una forma como se llega a la fiesta; con toda esa hermandad, con todas esas energías, con todos esos instrumentos mitológicos o canciones mitológicas, [...] pero al mismo tiempo yo estoy queriendo representar ahi como que... es una forma de protestar también [...] protestar con fiesta, con canciones, con conocimiento » (entretien avec Brus Rubio Churay, 6-7 mars 2019).

36. Dans le registre intermédiaire, une femme porte une calebasse avec une substance fumante. Brus Rubio Churay assure qu'il s'agit de piments piquants qui sont utilisés chez les Murui-Muina pour provoquer un effet chamanique, pour la purification des maisons.

37. Dans le registre intermédiaire, il y a une scène composée de deux hommes et d'une femme. Il s'agit de deux zaraiki qui interagissent avec une femme vêtue d'une robe verte. Cette femme porte une calebasse remplie de jus de manioc (manicuera). Dans cette scène, Brus Rubio Churay représente la deuxième partie du rituel de remise du gibier au cours duquel les femmes offrent du manicuera aux invités afin de les purifier (Figure 6). 
Nous avons vu également que de nombreux indices du tableau permettent d'afficher les identifications propres au rite et à la condensation rituelle. Pour représenter une danse rituelle, le peintre figure les objets rituels, les bâtons ou les feuilles de palmier qui permettent de reconnaître les différents groupes auxquels s'identifient les chanteurs-danseurs, tandis que, pour prendre un autre exemple, les calebasses de manioc ou de piment évoquent les actions de purification. Cependant, d'autres éléments, apparemment étrangers au rite - comme le palais présidentiel du Pérou, le livre ouvert de l'étudiant amérindien ou encore les papiers qui représentent les demandes adressées à l'État -, mettent en scène une condensation de relations contradictoires qui participent d'une façon plus profonde à l'action rituelle.

Finalement, la condensation rituelle n'est pas simplement « représentée » : l'artiste crée un dispositif dans lequel l'observateur est partie prenante du rite. Cette projection l'intègre dans le jeu des identifications : il devient le maître du rituel, auquel s'adresse le zaite. De plus, le peintre ne se limite pas à représenter le rite. Il l'utilise plutôt comme une boîte à outils destinés à la représentation. Pour ce faire, l'artiste mobilise un type de réflexivité épistémique au travers duquel il déchiffre la logique de construction du rite. En reprenant dans son tableau la structure du rite, il met en place des relations contradictoires et simultanées entre l'observateur et la communauté représentée, tout comme le rituel met en place des relations simultanées et contradictoires entre les participants indigènes. La invitación y llegada con fuerza n'est donc ni une action rituelle à part entière, ni une simple figuration de celle-ci; le tableau est placé sur un plan intermédiaire entre la danse et le rite. Reprenant la très pertinente formule de Careri, on pourrait dire que cette image est « une partie intégrant un réseau où les croyances et l'art ont des rôles spécifiques et des fonctions essentielles » (Careri 2003). *

* Manuscrit reçu en mai 2020, accepté pour publication en avril 2021.

\section{Références citées}

AdELl Nicolas

2016 «Panser les rituels. Parcours de rituels secrets dans l'espace public » [en ligne], ethnographiques.org, 33, https://www.ethnographiques.org/2016/Adell, consulté le 20/07/21.

BAL Mieke

1978 « Mise en abyme et iconicité », Littérature, 29, p. 116-128.

BATESON Gregory

1990 [1936] Naven « un ceremonial Iatmul ». Estudio de los problemas sugeridos por una visión compuesta de la cultura de una tribu de Nueva Guinea obtenida desde tres puntos de vista, Ediciones Jucar, Madrid. 
Représenter « avec » le rite : réflexivité et danse rituelle dans un tableau murui-muina

Belaunde Luisa Elvira

2011 « Visión de espacios en la pintura del sheripiari asháninka Noe Silva Morales », Mundo Amazónico, 2, p. 365-378.

CARERI Giovanni

2003 « Aby Warburg: rituel, pathos formel et forme intermédiaire », L'Homme, 165 (janvier-mars), p. 41-76.

Chaumeil Jean-Pierre

1994 «Los Yagua », in Fernando Santos Granero et Frederica Barclay (dir.), Guía Etnográfica de la Alta Amazonía, Vol. 1. Mai huna. Yagua. Ticuna, Institut français d'études andines (Travaux de l'IFEA, 81)/Flacso Ecuador, Lima.

2000 [1983] Voir, savoir, pouvoir. Le chamanisme chez les Yagua de l'Amazonie péruvienne, Georg Editeur, Genève.

CORTÉs GARZÓN Liliana

2015 Amazónicos: un estudio de pintores amazónicos actuales, thèse de doctorat en histoire de l'art et musicologie, Universidad Autonoma de Barcelona, Barcelona.

Descola Philippe

1993 « Les affinités sélectives. Alliance, guerre et prédation dans l'ensemble jivaro », L'Homme, 33 (126), p. 171-190.

Fausto Carlos et Eduardo Viveiros de CASTRo

1993 «La puissance et l'acte. La parenté dans les basses terres d'Amérique du Sud », L'Homme, 33 (126), p. 141-170.

Fdili Alaoui Sarah

2012 Dance Gesture Analysis and Visual Feedback based on Physical Models: Contributions of Movement Qualities in Whole Body Interaction, thèse de doctorat en informatique, Université Paris Sud - Paris XI, s.l.

Foucault Michel

1966 Les mots et les choses. Une archéologie des sciences humaines, Gallimard, Paris. GARCIA Oscar Ivan

2016 «Danzando fakariya: los bailes uitoto como modelo de organización social en la Amazonía », Bulletin de l'Institut français d'études andines, 45, p. 39-62.

2018 Rafue Ite! Ethnographie d'un bal rituel amazonien (Murui-Muina-Uitoto, Amazonie colombienne), thèse de doctorat en anthropologie sociale et ethnologie, EHESS, Paris.

2019 «Cantar para restituir el territorio: análisis comparativo de las artes de la memoria tukano y murui-muina (uitoto) », Alternativa. Revista de Estudios Rurales, 5 (9), http://ppct.caicyt.gov.ar/index.php/alter-nativa/article/view/16966, consulté le 20/07/21.

Gombrich Ernst Hans et Fritz SAXL

2015 [1970] Aby Warburg. Une biographie intellectuelle, traduit par Lucien d'Azay, Klincksieck, Paris.

Gombrich Ernst Hans et Guy Durand

1987 [1956] L'art et l'illusion. Psychologie de la représentation picturale, Gallimard, Paris.

Goulard Jean-Pierre

2010 «El Noroeste amazónico en perspectiva: una lectura desde los siglos V-VI hasta 1768 », Mundo Amazónico, 1 (enero), p. 183-214. 
Houseman Michael et Carlo Severi

2009 Naven ou le donner à voir essai d'interprétation de l'action rituelle, CNRS Éditions, Paris.

Humphrey Caroline et James LaidLaw

2004 The Archetypal Actions of Ritual. A Theory of Ritual Illustrated by the Jain Rite of Worship, Clarendon Press, Oxford.

KinTSURACHVILI Médéa

2010 « Les jeux et les enjeux de la mise en abyme », ANADISS, 2 (10), p. 115-132.

LABAN Rudolf

1994 La maîtrise du mouvement, traduit par Jacqueline Challet-Haas et Marion Bastian, Actes Sud (L'art de la danse), Paris.

Luna Luis Eduardo et Pablo Amaringo

1999 Ayahuasca Visions the Religious Iconography of a Peruvian Shaman, North Atlantic Books, Berkeley.

MAuss Marcel

1936 «Les techniques du corps », Journal de psychologie, 32 (3-4), p. 271-293.

McNeill David, Laura L. Pedelty et Elena T. Levy

1990 «Speech and gesture», in Geoffrey R. Hammond (dir.), Cerebral control of speech and limb movements, North-Holland (Advances in psychology, 70), Amsterdam, p. 203-256.

PINEDA-CAMACHO Roberto

2000 Holocausto en el amazonas. Una historia social de la Casa Arana, Planeta Colombiana Editorial, Santafé de Bogotá.

ROZENBERG Guillaume

2011 «Magie du rituel, démon de la réflexivité », L'Homme, 198-199, p. 277-299.

TAussig Michael

1991 Shamanism, Colonialism and the Wild Man. A Study in Terror and Healing, University of Chicago press, Chicago/Londres.

TAYLOR Anne-Christine

2009 «Métagermanité et affinité potentielle: la relation de mariage en Inde et en Amazonie », Ateliers d'anthropologie [en ligne], 33, https://journals.openedition.org/ateliers/8193, consulté le 20/07/21. DOI : 10.4000/ateliers.8193

Viveiros de CASTRo Eduardo

2009 Métaphysiques cannibales. Lignes d'anthropologie post-structurale, Presses universitaires de France (MétaphysiqueS), Paris.

Yluia María Eugenia

2009 «De la maloca a la galería: la pintura sobre llanchama de los boras y huitotos de la Amazonía peruana », Illapa Mana Tukukuq, 6, p. 95-108, https://revistas. urp.edu.pe/index.php/Illapa/article/view/1034, consulté le 20/07/21. DOI : 10.31381/illapa.v0i6.1034 